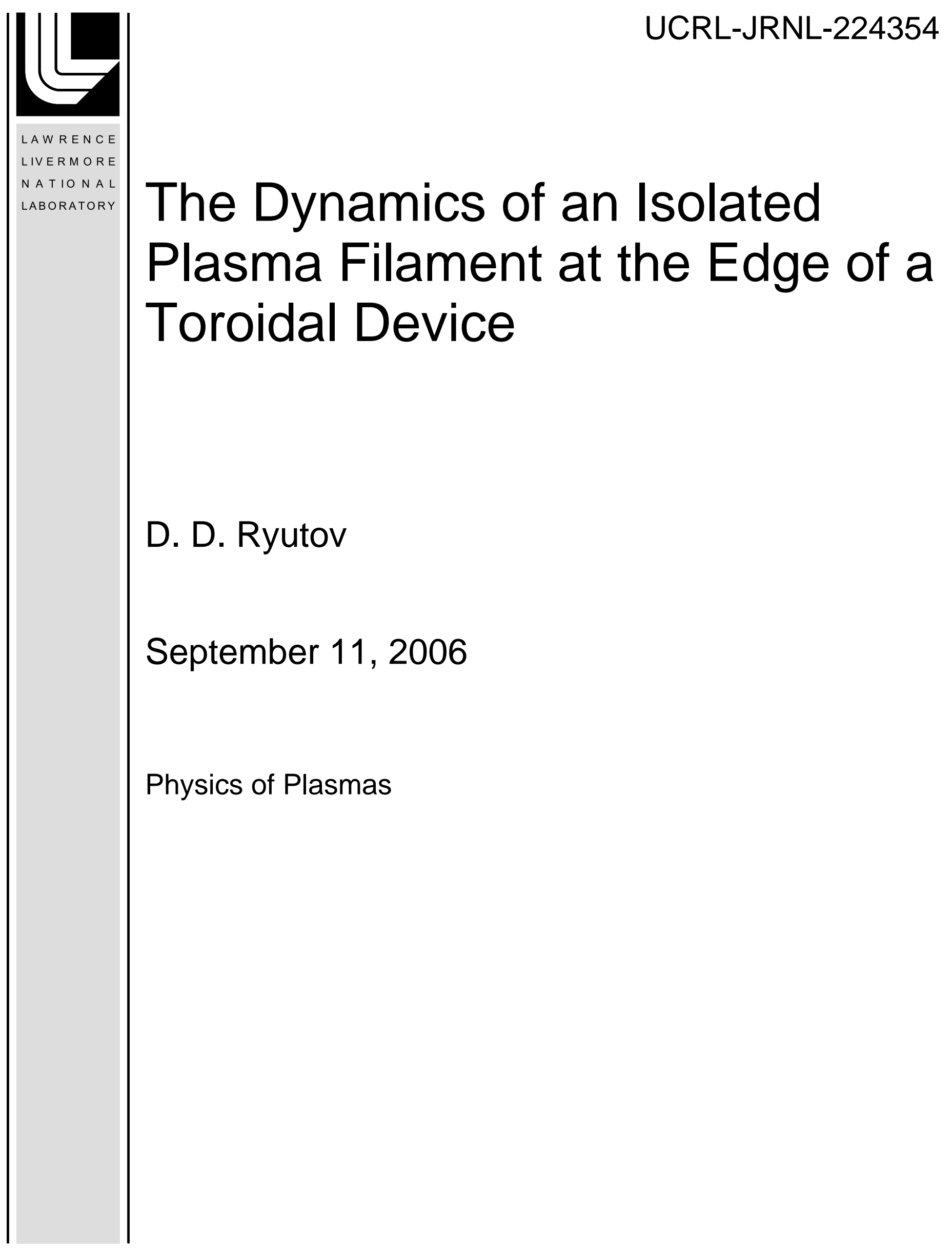


This document was prepared as an account of work sponsored by an agency of the United States Government. Neither the United States Government nor the University of California nor any of their employees, makes any warranty, express or implied, or assumes any legal liability or responsibility for the accuracy, completeness, or usefulness of any information, apparatus, product, or process disclosed, or represents that its use would not infringe privately owned rights. Reference herein to any specific commercial product, process, or service by trade name, trademark, manufacturer, or otherwise, does not necessarily constitute or imply its endorsement, recommendation, or favoring by the United States Government or the University of California. The views and opinions of authors expressed herein do not necessarily state or reflect those of the United States Government or the University of California, and shall not be used for advertising or product endorsement purposes. 


\title{
THE DYNAMICS OF AN ISOLATED PLASMA FILAMENT \\ AT THE EDGE OF A TOROIDAL DEVICE
}

\author{
D.D. Ryutov
}

Lawrence Livermore National Laboratory, Livermore, CA 94551

\begin{abstract}
The dynamics of an isolated plasma filament (an isolated blob) in the far scrapeoff layer (SOL) and the wall of a toroidal device is described, with a proper averaging of the geometrical parameters as well as plasma parameters along the filament. The analysis is limited to the magnetohydrodynamic description. The effects of the finite resistivity and anchored ends are also discussed.
\end{abstract}

\section{INTRODUCTION}

A number of experiments have shown that, at the periphery of a toroidal plasma, often beyond the main scrape-off layer (SOL), there may exist filamentary plasma structures which are strongly elongated along field lines and have small transverse crosssections (e.g., [1-3], and references therein). Their significance is related to the fact that, propagating into the far SOL, they may hit the tokamak wall in the area that might not be designed for accommodating high heat loads. Their dynamics is interesting also from the basic plasma physics standpoint. In this note we consider some aspects of this latter, basic side of the problem. The structures that we consider are often called "blobs" (after the important paper [4]), this term reflecting their shape in the poloidal cross-section. We will use this term interchangeably with "filaments."

In this note we consider mostly an "isolated filament," meaning by that a filament that satisfies the following two conditions: 1) The plasma density surrounding the 
filament is much smaller than the filament density and can therefore be neglected and, 2) The both ends of the filament are not in contact with any material surfaces. We do not discuss the formation of isolated blobs, assuming that they have somehow emerged from the main SOL plasma. One of the plausible mechanisms of formation of isolated blobs may be an explosive instability considered in Ref. [5]. In addition to the blobs with free ends, we consider also the situation where one or both ends of the blob are anchored, and the filament dynamics is governed by resistive effects.

In the past, numerous aspects of the blob physic have been looked at. In Ref. [6], the dynamics of an isolated blob that is in contact with the divertor plate has been qualitatively analyzed; the role of the X-point was taken into account in the form of a "heuristic" boundary condition [7]. In Ref. [8], also on the qualitative level, the evolution of isolated blobs in the divertor region was considered, with both the sheath boundary condition at the divertor plate and the "heuristic" boundary condition near the X-point imposed. In Ref. [9], the blobs were analyzed on the basis of the MHD vorticity equation. An extensive study of the blob dynamics based on the vorticity equation was offered in Ref. [10]. Viscous effects and the parallel dynamics and heat conduction were taken into account. Other aspects of the blob theory have been briefly summarized in Ref. [11].

In the present paper, based entirely on the MHD equations, we concentrate on the geometrical features of isolated filaments associated with the facts that the field line curvature and other parameters of the magnetic field may vary substantially along the long filament. To set the stage to this study, we repeat a simple derivation presented in Ref. [6] to illustrate the blob dynamics. As was shown in Refs. [4, 6] the cross-field motion of the blob is determined from the condition that the cross-field current generated 
by the curvature drift be compensated by the polarization current driven by the plasma inertia. To evaluate an acceleration of an insulated filament, one can note (e.g., [5]), that the curvature-driven current density can be estimated as $c p / R B$, and the polarization current density as $c \rho \ddot{\xi}_{\perp} / B$ (in these estimates, $p$ and $\rho$ are the plasma pressure and density, respectively, $B$ is the magnetic field, $R$ is the major radius, $c$ is the velocity of light, and $\ddot{\xi}_{\perp}$ is the radial acceleration of the filament; we use CGS Gaussian system of units). By equating the two current densities, one obtains the following rough estimate:

$$
\ddot{\xi}_{\perp} \sim \frac{p}{R \rho}
$$

The sign of this acceleration is such that the filament moves away from the plasma on the low-field side and towards the plasma on the high-field side of a torus.

When evaluating the acceleration in Eq. (1), we tacitly assumed that the filament is short-enough, so that the curvature radius is a well-defined quantity. However, if the filament is long, so that the curvature substantially varies along its length, the question arises as to how one should average the curvature, as well as the pressure and the density. The present note provides an answer to this question within the MHD approximation.

We consider the blob as a well-defined object, clearly separated from its environment. Such blobs have been observed experimentally (see the images in Refs. [1][3]) and often demonstrate a remarkable cohesion in the course of their propagation towards the walls. To address this feature, we assume that the deformation of the crosssection of the filament is suppressed by some un-specified viscous forces and evaluate the viscosity which would be sufficient to provide cohesion. The analysis of the viscous force in Ref. [10] indeed shows that its presence suppresses the internal dynamics inside the filament. On the other hand, if the viscosity is small, and the filament changes its 
cross-field shape in the course of the radial motion, our results would correspond to the motion of some "median" line of the filament.

\section{BASIC EQUATIONS AND GEOMETRICAL FRAMEWORK}

We use the following set of equations:

$\rho \frac{d \mathbf{v}}{d t}=-\nabla p+\frac{\boldsymbol{j} \times \boldsymbol{B}}{c}+\boldsymbol{f}_{\text {visc }}$

$\frac{\partial \boldsymbol{B}}{\partial t}=\nabla \times \mathbf{v} \times \boldsymbol{B}$

$\nabla \times \boldsymbol{B}=\frac{4 \pi}{c} \boldsymbol{j}$

where $\boldsymbol{j}$ is the current density and $\boldsymbol{f}_{\text {visc }}$ is a viscous force.

The plasma at the edge of fusion devices, especially in the far SOL, has a low pressure. This allows one to neglect the magnetic field perturbations in the course of the displacement of the flux tube and set $\partial \boldsymbol{B} / \partial t=0$. Then Eq. (3) yields in a standard way:

$\mathbf{v} \times \boldsymbol{B}=-(1 / c) \nabla \varphi$

We have written the right-hand side (rhs) in a way that allows one to associate the function $\varphi$ with the electrostatic potential. Eq. (5) shows that $\varphi$ is constant along the field lines, i.e., the potential is a function of the field line. So, the cross-field displacement of a certain element of a field line determines the displacement over its whole length. As is well known (e.g., [7]), this means that the allowed displacements bring one field line (together with particles populating it) to another field line.

It is convenient to present a displacement of a certain field line as a superposition of displacement $\xi_{n}$ normal to the poloidal flux surface, and displacement $\xi_{g}$ lying within the flux surface but perpendicular to the field line (the subscript " $g$ " stands for "geodesic"). These two displacements are perpendicular to each other and to the field 
line. If one introduces displacement $\xi_{\perp 0}=\left(\xi_{n 0}, \xi_{g 0}\right)$ in a certain reference point on the field line, then displacement in any other point can be found from purely geometric considerations. For the normal displacement (Fig. 1) the result is:

$\xi_{n}=\xi_{n 0} \frac{B_{p 0} R_{0}}{B_{p} R}$

This equation follows from the condition that the poloidal flux between the two neighboring flux surfaces is constant. The subscript " 0 " here designates the quantities at the reference point, $B_{p}$ is the poloidal component of the magnetic field, and $R$ is the major radius, Fig. 1.

For the geodesic displacement the result is somewhat more complex. The derivation is presented in Appendix 1, where it is shown that

$\xi_{g}=\left[\xi_{g 0}-\xi_{n 0} Q\right]\left(\frac{B_{P} R}{B}\right)\left(\left(\frac{B_{0}}{B_{p 0} R_{0}}\right)\right)$

where the quantity $Q$ is introduced in the Appendix I (Eq. (A.4)) and can be called an "average shear." In particular, it determines the ellipticity $E$ of the flux tube that has a circular cross-section in the reference point. By $E$ we mean the ratio of the major semiaxis to the minor semi-axis. In the case of a strong shear, the ellipticity can be presented as

$$
E=Q^{2}
$$

\section{GLOBAL MOTION OF THE FLUX TUBE}

For a large-enough viscous force, any cross-section of the flux-tube will move as a whole, without a mutual slippage of the liquid elements occupying this cross-section. So, for a large-enough viscous force, displacement of the filament can be characterized by a single vector $\xi_{\perp}$, the same for all the pieces of a given cross-section. In other words, 
the shape of the displaced flux-tube can be characterized by a displacement vector $\xi_{\perp}(s, t)$, which is a function of a single spatial coordinate $s-$ the distance along the field line - and the time $t$.

On the other hand, as the viscous force is a force of mutual friction of various elements inside the plasma, the net viscous force acting in a certain cross-section of the flux tube is zero:

$$
\int f_{v i s c} d S=0
$$

Our further plan consists in using Eq. (2) to find the cross-field current, imposing a constraint (9) and, from the current-continuity equation integrated over the length of the flux-tube, finding the acceleration in the reference point (and, by virtue of Eqs. (6) and (7), in any point of the flux tube). From Eq. (2) we obtain:

$\boldsymbol{j}_{\perp}=-\frac{c \rho \ddot{\boldsymbol{\xi}} \times \boldsymbol{B}}{B^{2}}+c \nabla \times\left(\frac{p \boldsymbol{B}}{B^{2}}\right)-2 c p\left(\frac{\boldsymbol{B} \times \nabla B}{B^{2}}\right)+c \frac{\boldsymbol{f}_{\text {visc }} \times \boldsymbol{B}}{B^{2}}$

We have used an identity (valid for the vacuum magnetic field): $\nabla p \times \boldsymbol{B} / B^{2}=\nabla \times\left(p \boldsymbol{B} / B^{2}\right)-2 p(\boldsymbol{B} \times \nabla B) / B^{3}$. Now we want to use Eq. (9) and need to integrate Eq. (8) over a cross-section of the filament. In every cross-section, one can introduce a coordinate frame with the axes oriented along the two mutually orthogonal directions " $n$ " and " $g$ " (see discussion before Eq. (6)). The corresponding coordinates in every cross-section are $x_{n}$ and $x_{g}$, respectively, measured from some median field line in the flux tube (as we shall see, its choice does not matter). When performing the integration, we notice that the second term in the rhs of Eq. (10) is a sum of the derivatives $\partial / \partial x_{n}, \partial / \partial x_{g}$ and yields zero when integrated over the cross-section (over $d S=d x_{n} d x_{g}$ ). We further note that the displacement is uniform over the cross-section and 
the parameters of the magnetic field vary very little over the cross-section of a thin tube, so the integration in the first and the third terms in (10) will be reduced to the integrals $\int \rho d S$ and $\int p d S$. With these notions made, one finds:

$\int \boldsymbol{j}_{\perp} d S=-\frac{c \ddot{\boldsymbol{\xi}} \times \boldsymbol{B}}{B^{2}} \int \rho d S-2 c\left(\frac{\boldsymbol{B} \times \nabla B}{B^{3}}\right) \int p d S$

In all these calculations we retained only the leading-order terms in the small parameter $a / R$, where $a$ is the filament radius. In particular, we neglected the variation of the magnetic field $B$ across the cross-section of the flux-tube.

Now we use the current continuity equation which can be written as

$\nabla \cdot \boldsymbol{j}_{\perp}=-B \frac{\partial}{\partial s} \frac{j_{\|}}{B}$,

where we have $j_{\|}=j_{\|}\left(s, x_{n}, x_{b}\right)$. By multiplying the 1.h.s. of Eq. (12) consecutively by $x_{n}$ and $x_{g}$, and performing integration over the cross-section (i.e., over $d S=d x_{n} d x_{g}$ ), one finds:

$\frac{1}{B} \int j_{n, g} d S=\frac{\partial}{\partial s}\left(\frac{1}{B} \int x_{n, g} j_{\|} d S\right)$

Then, integrating this equation over $d s$ and taking into account that $j_{\|}$is zero at both ends of the isolated filament, we find

$\int \frac{d s}{B} \int j_{n, g} d S=0$

This result is indeed independent on the particular choice of the median field line.

Using Eqs. (11) and (14) we then obtain

$\int d s \frac{\ddot{\xi}_{n, g}}{B^{2}} \int \rho d S=\mp 2 \int d s \frac{(\nabla B)_{n, g}}{B^{3}} \int p d S$ 
The signs are determined by our convention regarding the local triplet of the unit vectors: the first is a unit vector along the magnetic field, the second is the external normal to the flux surface, and the third (in the $g$ direction) is a vector product of the first and the second.

Finally, substituting Eq. (6) into Eq. (13), we find the following result for the acceleration of the reference point:

$$
\begin{aligned}
& \ddot{\xi}_{n 0}=-\frac{2}{B_{p 0} R_{0}}\left[\int d s \frac{(\nabla B)_{n}}{B^{3}} \int p d S\right]\left[\int \frac{d s}{B^{2} B_{p} R} \int \rho d S\right]^{-1} \\
& \ddot{\xi}_{g 0}+\ddot{\xi}_{n 0} B_{P 0} R_{0}\left[\int \frac{Q B_{p} R d s}{B^{3}}\left(\int \rho d S\right)\right]\left[\int \frac{B_{p} R d s}{B^{3}} \int \rho d S\right]^{-1} \\
& =\frac{2 B_{p 0} R_{0}}{B_{0}}\left[\int d s \frac{(\nabla B)_{g}}{B^{3}} \int p d S\right]\left[\int \frac{B_{p} R d s}{B^{3}} \int \rho d S\right]^{-1}
\end{aligned}
$$

This result is quite general in that we have not made any assumptions regarding the spatial dependence of the pressure and density inside the flux tube. Note that the sign of $(\nabla B)_{n}$ can change along the filament. This happens, for example, when the filament encompasses both the low-field side and a high-field side of a tokamak with a single-null divertor. Then, the overall direction of normal acceleration (to the wall or away from the wall) is determined by the proper weighing of the plasma parameters over the flux tube, as described by Eq. (16).

In some cases, it is more convenient to switch in Eqs. (16), (17) to integration along the poloidal circumference, by using the identity $d s=\left(B / B_{p}\right) d l$. This yields:

$$
\ddot{\xi}_{n 0}=-\frac{2}{B_{p 0} R_{0}}\left[\int d l \frac{(\nabla B)_{n}}{B^{2} B_{p}} \int p d S\right]\left[\int \frac{d l}{B B_{p}^{2} R} \int \rho d S\right]^{-1}
$$




$$
\begin{aligned}
& \ddot{\xi}_{g 0}+\ddot{\xi}_{n 0} B_{P 0} R_{0}\left[\int \frac{Q R d l}{B^{2}}\left(\int \rho d S\right)\right]\left[\int \frac{R d l}{B^{2}} \int \rho d S\right]^{-1} \\
& =\frac{2 B_{p 0} R_{0}}{B_{0}}\left[\int d l \frac{(\nabla B)_{g}}{B_{p} B^{2}} \int p d S\right]\left[\int \frac{R d l}{B_{p} B^{2}} \int \rho d S\right]^{-1}
\end{aligned}
$$

\section{EXAMPLES}

Consider first a situation where the shear effect is insignificant, and the second term in the lhs of Eq. (17) can be neglected. Then, the direction of the motion of the flux tube is determined by the properly weighted gradients of the magnetic field $(\nabla B)_{n}$ and $(\nabla B)_{g}$. If the fluxtube is situated at the low-field side, as shown in Fig. 2, the normal gradient is directed away from the plasma, and the filament moves toward the wall. The geodesic acceleration (which is roughly equal to the poloidal acceleration) may change sign depending on whether the flux tube occupies predominantly the upper or the lower part of the cross-section. In the first case, the fluxtube near the equatorial plane will move upward, whereas in the second case it will move downward (Fig. 2 a,b).

If the pressure and density are both uniform from one end of the tube to another, the result can be presented as:

$$
\ddot{\xi}_{n 0}=-\frac{1}{B_{p 0} R_{0}}\left[\int d s \frac{(\nabla B)_{n}}{B^{3}}\right]\left[\int \frac{d s}{B^{2} B_{p} R}\right]^{-1} \frac{4 W}{3 M} ; \quad \ddot{\xi}_{g 0}=\frac{B_{p 0}}{B_{0}}\left[\int d s \frac{(\nabla B)_{g}}{B^{3}}\right]\left[\int \frac{B_{p} d s}{B^{3}}\right]^{-1} \frac{4 W}{3 M}
$$

where $W$ is the thermal energy of the plasma in the flux tube, $M$ is the mass of that plasma, and integrations are taken over the segment filled with the plasma. In this case the ratio of the normal and tangential forces is independent on the plasma parameters and is determined by purely geometric factors. The same is true if the filament is short, so that the parameters of the magnetic field do not vary substantially over the length of the 
plasma-filled segment. Then, one can take the magnetic field out of the integrals in Eqs. (18) to obtain:

$\ddot{\xi}_{n}=-\frac{(\nabla B)_{n}}{B} \frac{4 W}{3 M} ; \quad \ddot{\xi}_{g}=-\frac{(\nabla B)_{g}}{B} \frac{4 W}{3 M}$

We have dropped the subscript " 0 " because all the points of a short segment can be characterized by the same acceleration. Note that we do not need here the assumption of the uniformity of the plasma parameters over the length. Eq. (19) is a quantitative generalization of the estimate (1).

In the case where shear is substantial, in particular, if one end of the flux tube comes close to the X-point (Fig. 2c), it is the shear term that determines the poloidal displacement. In this case, one can neglect the r.h.s. in Eq. (17'). For the situation shown in Fig. 2c, the shear term is dominated by the contribution of the vicinity of the X-point and is positive. For a large average shear, the poloidal displacement in the observation point can be much larger than the normal displacement.

As has already been mentioned, for the large shear the shape of the cross-section of the flux-tube can experience substantial changes along the field line. In general, Eqs. (6) and (7) allow one to predict the shape of the flux tube cross-section based on the observations in a single toroidal location.

\section{APPLICABILITY LIMITS}

If the flux tube starts with a zero velocity, then the ratio of the normal and geodesic accelerations is also the ratio of the corresponding velocities. If the flux-tube has traveled radially a distance $D$, then the characteristic velocity determined from Eq. (1) [or (19)] is

$\mathrm{v} \sim \mathrm{v}_{T i} \sqrt{D / R}$ 
Let us now estimate the magnitude of the viscosity required for suppressing a mutual slippage of the elements of the flux tube. To maintain the cohesion of the filament, the friction force must be greater than the driving force which is of order of $p / R$ (per unit volume). The friction force is $\rho v \delta v / a^{2}$, where $v$ is the kinematic viscosity and $\delta v$ is the velocity variation over the flux-tube cross-section. We want the velocity variation $\delta \mathrm{v}$ to be much less than the average velocity $\mathrm{v}(20)$ reached when the fluxtube got displaced by the distance of order of its radius $a$, and, at the same time, the friction force to be greater than the driving force, i.e., $\rho v \delta \mathrm{v} / a^{2}>p / R$. This yields the following constraint on the flux-tube radius: $a<\left(v^{2} \mathrm{R} / \mathrm{v}_{\mathrm{Ti}}^{2}\right)^{1 / 3}$. Assuming that $v$ is of order of the Bohm kinematic viscosity, $v \sim r_{L i} \mathrm{v}_{T i}$, one finds: $a / r_{L i}<\left(R / r_{L i}\right)^{1 / 3}$. Typically, this limits $a$ to the scale of 10-30 ion gyroradii.

If viscosity is insufficient (as is the case for thick flux tubes), then various "subfilaments" constituting the initial filament, will accelerate at a different rate, and initially well defined flux-tube will get dispersed in the radial and toroidal directions (the size of its cross-section will become of order of the distance traveled).

Consider now our assumption of a low plasma beta. We have neglected the magnetic field perturbations by the plasma currents associated with the motion of the flux tube. Now we evaluate this perturbation. The main contribution to it comes from the parallel current $j_{\|}$, as it is by a factor of $l / a$ (where $l$ is the length of a filament) larger than the perpendicular current. An estimate of the parallel current that follows from Eqs. (11), (12) is: $j_{\|} \sim c p l / a R B$. This current generates the magnetic field that has a component $\delta B_{\perp} \sim 4 \pi l p / B R$, perpendicular to the main magnetic field and causes a displacement of field lines in the filament from their initial positions by the distance $\sim \delta B_{\perp} l / B$. Imposing 
the constraint that this displacement be less than the filament radius $a$, we obtain the following constraint on the plasma beta: $\beta<a R / l^{2}$. This condition is rather restrictive. One should however remember that the parallel current may actually be smaller than $j_{\|} \sim c p l / a R B:$ this estimate assumes that there exists a significant divergence of the cross-field current, whereas this is not necessarily the case. In particular, if the filament is not too long, so that he curvature can be considered as constant, then the cancellation of the curvature-driven current and the polarization current occurs in every cross-section, and the parallel current becomes much less than $j_{\|} \sim c p l / a R B$. Here we assume that the mass is also uniformly distributed along the filament: if some part of it is too heavily loaded, the local cancellation becomes impossible.

\section{SHEATH RESISTANCE}

For the situation where the filament has reached the zone in the shadow of a poloidal limiter, either deliberately introduced, or formed by some naturally present structural elements, one (or both) of its ends come in contact with the limiter surface (Fig. 3). For a conducting limiter, a considerable reduction of the polarization electric field may occur, leading to a slowing down of the filament motion. However, because of a finite resistance of the sheath at the plasma-wall interface, the reduction of the electric field is only partial, meaning that there is no perfect line-tying at the wall. This observation dates back to a paper of Kunkel and Guillory []. In the problem of the blob propagation the appropriate boundary condition was used, in particular in Refs. [ - ].

In the formal description of this phenomenon, we have to allow for a current flow to the limiter and impose the boundary condition relating the current and the potential in this zone. Instead of Eq. (), we will have now: 
$\int \frac{d s}{B} \int j_{n, g} d S=\left.\frac{1}{B_{0}} \int x_{n, g} j_{\|}\right|_{e n d} d S$

We consider the situation where the limiter surface is normal to the toroidal magnetic field, as shown in Fig. 3. In this case, one can use the following current-voltage characteristics (Cf. Refs. [9,10,15]):

$j_{\|}=j_{s a t} \frac{e\left(\varphi-\varphi_{f}\right)}{T_{e}}$

where $j_{s a t} \sim e n \mathrm{v}_{T i}$ is the ion saturation current density. We use the current-voltage characteristic in a linearized form, assuming that the current is smaller than the ion saturation current. In Eq. (22), $\varphi$ is the plasma potential with respect to the grounded limiter, and $\varphi_{f}$ is the floating potential.

For the filament that is in contact with the limiter, we use, as a reference point, its intersection with the limiter. The velocity of the filament at this point is determined by the equations:

$$
\dot{\xi}_{n 0}=\frac{c E_{g 0}}{B_{0}} ; \dot{\xi}_{g 0}=-\frac{c E_{n 0}}{B_{0}}
$$

and the plasma potential, accordingly, is

$$
\varphi=\frac{B_{0}}{c}\left(x_{g} \dot{\xi}_{n}-x_{n} \dot{\xi}_{g}\right)+C
$$

The integration constant is determined from the condition that the net current through the filament is zero (for the other end not in contact with material surface, Fig. 3; otherwise, the constant has to be determined by matching the current at two ends):

$$
C=\left(\int \frac{j_{s a t} \varphi_{f}}{T_{e}} d S+\frac{B_{0} \dot{\xi}_{n}}{c} \int \frac{j_{s a t} x_{g}}{T_{e}} d S-\frac{B_{0} \dot{\xi}_{g}}{c} \int \frac{j_{s a t} x_{n}}{T_{e}} d S\right)\left(\int \frac{j_{s a t}}{T_{e}} d S\right)^{-1}
$$


We assume that the density of the filament falls off from the central line rapidly enough to make integrals in this equation convergent. After this preparatory work, we substitute the current (22) into Eq. (21), with the potential related to the velocity of the filament by Eq. (25). This yields:

$$
\begin{aligned}
& \left.\int j_{\|}\right|_{e n d} x_{g} d S=\frac{e B_{0}}{c} \dot{\xi}_{n} \int \frac{x_{g}^{2} j_{s a t}}{T_{e}} d S-\frac{e B_{0}}{c} \dot{\xi}_{g} \int \frac{x_{g} x_{n} j_{s a t}}{T_{e}} d S+ \\
& \frac{e B_{0} \dot{\xi}_{n}}{c}\left(\int \frac{x_{g} j_{s a t}}{T_{e}} d S\right)^{2}\left(\int \frac{j_{s a t}}{T_{e}} d S\right)^{-1}-\frac{e B_{0} \dot{\xi}_{g}}{c}\left(\int \frac{x_{g} j_{s a t}}{T_{e}} d S\right)\left(\int \frac{x_{n} j_{s a t}}{T_{e}} d S\right)\left(\int \frac{j_{s a t}}{T_{e}} d S\right)^{-1}+ \\
& e\left(\int \frac{x_{g} j_{s a t}}{T_{e}} d S\right)\left(\int \frac{j_{s a t} \varphi_{f}}{T_{e}} d S\right)\left(\int \frac{j_{s a t}}{T_{e}} d S\right)^{-1}-e\left(\int \frac{x_{g} j_{s a t} \varphi_{f}}{T_{e}} d S\right) \\
& \int j_{\|} \|_{\text {end }} x_{n} d S=-\frac{e B_{0}}{c} \dot{\xi}_{g} \int \frac{x_{n}^{2} j_{s a t}}{T_{e}} d S+\frac{e B_{0}}{c} \dot{\xi}_{n} \int \frac{x_{g} x_{n} j_{s a t}}{T_{e}} d S- \\
& \frac{e B_{0} \dot{\xi}_{g}}{c}\left(\int \frac{x_{n} j_{s a t}}{T_{e}} d S\right)^{2}\left(\int \frac{j_{s a t}}{T_{e}} d S\right)^{-1}+\frac{e B_{0} \dot{\xi}_{n}}{c}\left(\int \frac{x_{g} j_{s a t}}{T_{e}} d S\right)\left(\int \frac{x_{n} j_{s a t}}{T_{e}} d S\right)\left(\int \frac{j_{s a t}}{T_{e}} d S\right)^{-1}+ \\
& e\left(\int \frac{x_{n} j_{s a t}}{T_{e}} d S\right)\left(\int \frac{j_{s a t} \varphi_{f}}{T_{e}} d S\right)\left(\int \frac{j_{s a t}}{T_{e}} d S\right)^{-1}-e\left(\int \frac{x_{n} j_{s a t} \varphi_{f}}{T_{e}} d S\right)
\end{aligned}
$$

As it should be, this result is invariant with respect to the choice of a median field line (changing the origin in the $x_{n}, x_{g}$ plane).

Worth attention are the last two terms in the r.h.s. of Eqs. (26), (27). If they are non-zero, they represent additional drive for the average displacement of the filament, unrelated to curvature effects. They are the consequence of possible asymmetries in the temperature and density distribution across the filament and are related to the sheathdriven modes considered, e.g., in Ref. [16]. If these terms are present, whereas the curvature is absent, the filament would move with the velocity (that can be evaluated from Eqs. (26), (27)) $\dot{\xi}_{\perp} \sim c \varphi_{f} / a B$. The direction will be determined by the details of the temperature and density distribution. 
Assuming that the density and temperature distribution possess an inversion symmetry with respect to some central line, we delete the corresponding terms and end up with the following set of equations:

$$
\begin{aligned}
& \dot{\xi}_{n}\left(\int \frac{x_{g}^{2} j_{s a t}}{T_{e}} d S\right)=\frac{c}{B_{0}} \int d l \frac{(\nabla B)_{n}}{B^{2} B_{p}} \int p d S \\
& \dot{\xi}_{g}\left(\int \frac{x_{g}^{2} j_{s a t}}{T_{e}} d S\right)=-\frac{c}{B_{0}} \int d l \frac{(\nabla B)_{g}}{B^{2} B_{p}} \int p d S
\end{aligned}
$$

For a flux-tube with uniform parameters, and having a circular cross-section of a radius $a$ in the reference point, these equations reduce to

$$
\dot{\xi}_{n}=\frac{2 c T_{e} p}{a^{2} j_{s a t}} \int d l \frac{(\nabla B)_{n}}{B^{3} B_{p}}, \dot{\xi}_{g}=-\frac{2 c T_{e} p}{a^{2} j_{s a t}} \int d l \frac{(\nabla B)_{g}}{B^{3} B_{p}}
$$

\section{RESISTIVE BALLOONING}

It is easy to include in this description the effects of the finite plasma resistivity. This will be the parallel resistivity, because $j_{\|}$is much higher than $j_{\perp}$. The presence of the parallel current in the flux tube of a finite resistivity requires the presence of the parallel electric field and, therefore, the purely flute-like displacement (Eq. (6), (7)) becomes impossible. The plasma filament can now deviate from the magnetic field lines, the effect that can be called "resistive ballooning" (e.g., [13]). In the frame co-moving with the plasma filament, there will appear a time-varying magnetic field threading the flux tube in the direction perpendicular to the flux tube. Simple geometrical consideration shows that this field is:

$$
\delta \dot{B}_{n}=-\frac{1}{B_{p} R} \frac{\partial}{\partial s}\left(B_{p} R \dot{\xi}_{n}\right) ; \delta \dot{B}_{g}=-\frac{B_{p}}{B} \frac{\partial}{\partial s}\left(\frac{B}{B_{p}} \dot{\xi}_{g}\right) .
$$


In the approximation of a thin flux tube, this field can be considered as uniform over the cross-section of the tube.

The Faraday law, $\nabla \times \boldsymbol{E}=-(1 / c) \delta \dot{\boldsymbol{B}}$, then yields for the parallel electric field:

$$
\frac{\partial E_{\|}}{\partial x_{n}}=-\delta \dot{B}_{g} ; \quad \frac{\partial E_{\|}}{\partial x_{g}}=\delta \dot{B}_{n}
$$

For a uniform $\delta \boldsymbol{B}$, these equations can be readily integrated to yield:

$$
E_{\|}=-x_{n} \frac{B_{p}}{B} \frac{\partial}{\partial s}\left(\frac{B}{B_{p}} \dot{\xi}_{g}\right)+\frac{x_{g}}{B_{p} R} \frac{\partial}{\partial s}\left(B_{p} R \dot{\xi}_{n}\right)+C
$$

where the parameter $\mathrm{C}$ will be determined shortly. This parameter and the coefficients by which $x_{n}$ and $x_{g}$ are multiplied do not depend on $x_{n}, x_{g}$ in the thin-tube approximation. The current density is $j_{\|}=\sigma\left(x_{n}, x_{g}, s\right) E_{\|}$, where $s$ is the electrical conductivity. The net current through the flux-tube is zero, this allowing us to find $C$ :

$$
C=<x_{n}>\frac{B_{p}}{B} \frac{\partial}{\partial s}\left(\frac{B}{B_{p}} \dot{\xi}_{g}\right)-\frac{<x_{g}>}{B_{p} R} \frac{\partial}{\partial s}\left(B_{p} R \dot{\xi}_{n}\right)
$$

with

$$
<x_{n, g}>=\left(\int x_{n, g} \sigma d S\right) / \int \sigma d S
$$

We assume that the conductivity falls off rapidly enough outside the tube, so that the integrals in Eq. (36) converge.

The final step is to substitute the parallel current density into Eq. (13) and then use Eq. (11). This yields:

$$
\begin{aligned}
& \ddot{\xi}_{g} \int \rho d S+\frac{B^{2}}{c^{2}} \frac{\partial}{\partial s}\left\{\left(\int \sigma d S\right)\left[\frac{A_{n n} B_{p}}{B^{2}} \frac{\partial}{\partial s}\left(\frac{B}{B_{p}} \dot{\xi}_{g}\right)+\frac{A_{n g}}{B_{B} R} \frac{\partial}{\partial s}\left(B_{p} R \dot{\xi}_{n}\right)\right]\right\}+\frac{2(\nabla B)_{g}}{B} \int p d S=0, \\
& \ddot{\xi}_{n} \int \rho d S+\frac{B^{2}}{c^{2}} \frac{\partial}{\partial s}\left\{\left(\int \sigma d S\right)\left[\frac{A_{n g} B_{p}}{B^{2}} \frac{\partial}{\partial s}\left(\frac{B}{B_{p}} \dot{\xi}_{g}\right)+\frac{A_{n n}}{B B_{p} R} \frac{\partial}{\partial s}\left(B_{p} R \dot{\xi}_{g}\right)\right]\right\}+\frac{2(\nabla B)_{n}}{B} \int p d S=0,
\end{aligned}
$$


where

$$
A_{n n}=\left\langle x_{n}^{2}\right\rangle-\left\langle x_{n}\right\rangle^{2} ; A_{n g}=\left\langle x_{n} x_{g}\right\rangle-\left\langle x_{n}\right\rangle\left\langle x_{g}\right\rangle ; A_{n n}=\left\langle x_{g}^{2}\right\rangle-\left\langle x_{g}\right\rangle^{2}
$$

are defined analogously to Eq. (36). These coefficients do not depend on the choice of the median line in the flux tube.

General equations (37), (38) account for possible resistive effects in a complex geometry. In the high-conductivity limit, $\sigma \rightarrow \infty$, in order to keep the second term in the lhs comparable to the other terms, one has to impose a constraint (6). After that, by dividing equations (37), (38) by $B^{2}$ and integrating them along the flux tube, one eliminates the second term, and recovers Eqs. (14), (15). In other words, in the highconductivity limit, the resistive effects do not bring up anything particularly new to the flute-like solution (16), (17).

The situation changes dramatically if one or both ends of the filament are anchored, i.e., the displacements there is held zero. We do not discuss here possible mechanisms for anchoring, just look into its consequences. If we neglected the plasma resistivity, we would have to conclude that the flux-tube cannot move and stays at rest. However, accounting for even a small resistivity allows for resistive ballooning. As, at low resistivity, the corresponding motion is slow, one can neglect the first term in the 1.h.s. of Eqs. (37), (38). The remaining equations are easily integrable, although, in a general case, the result is very lengthy. As an example, we consider a flux-tube which is short compared to the connection length (but still long compared to $a$ ); in this case all the parameters of the magnetic field do not change significantly over the flux-tube length. The shape of the flux-tube cross-section also does not change. We assume that this is just a circle of a radius $a$. All the other parameters, $p, \rho$, and $\sigma$, are assumed to be constant 
over the whole volume of the flux tube. Under such conditions, Eqs. (37), (38) are reduced to:

$\partial^{2} \dot{\xi}_{n, g} / \partial s^{2}= \pm 4 D_{M} \beta(\nabla B)_{n, g}$.

For the flux-tube anchored at one end (say, $s=0$ ), this equation has a solution:

$\dot{\xi}_{n, g}= \pm 2 D_{M} \beta(\nabla B)_{n, g}\left(s^{2}-2 s l\right) / a^{2}$,

where $D_{M}=c^{2} / 4 \pi \sigma$ is the magnetic diffusivity. The time for the flux-tube end to move by the distance equal to the flux-tube radius is $\delta t \sim R a^{3} / l^{2} \beta D_{M}$. Then, evaluating the acceleration as $a / \delta t^{2}$, one finds that the inertial term in a full equations (37), (38) is negligible if $D_{M}<\mathrm{v}_{T i} a \sqrt{R a^{3}} / \beta l^{2}$, i.e., at low-enough resistivity. Note that the ratio $\dot{\xi}_{n} / \dot{\xi}_{g}=-(\nabla B)_{n} /(\nabla B)_{g}$ remains the same as the ratio of accelerations in the problem of the short filament with free ends.

When the filament deviates from the field line, the parallel plasma expansion along the field lines can lead to the change of the plasma density and pressure in any particular cross-section. In order this not to happen, the condition $l / a>\mathrm{v}_{T i} / \dot{\xi}_{\perp}$ must be satisfied, i.e., the flux tube must be long enough.

In summary: within the framework of a single-fluid hydrodynamics, we have derived expressions determining the dynamics of an isolated plasma filament at the periphery of a toroidal plasma, with a proper averaging of the magnetic field and plasma parameters along the filament. Both flute-like and resistive ballooning dynamics were considered. The cohesiveness of the filament motion was assumed to be provided by viscous forces which suppressed internal cross-field plasma motion. The results obtained can be of some interest in developing more complex models (beyond the single-fluid MHD), where the complex geometry of the magnetic field may also play significant role. 


\section{DISCUSSION}

We have considered the motion of a thin, low-beta, isolated plasma filament in the far scrape-off layer. For a filament of a substantial length, the parameters of the magnetic field (e.g., field line curvature) can vary significantly over the filament length, and the resulting motion is determined by some averaged (over the length) parameters of the field. We present such averaging procedures for an arbitrary toroidally-symmetric field. Although developed for the case where the displacement of every cross-section of the filament can be characterized by a single displacement vector (no slippage), the result may serve as a guidance for evaluating the average motion of a filament with internal slippage allowed. In a sense, our study is complementary to those of Refs. $[9,10]$, where the slippage was allowed but a simple model of the magnetic field was used.

Generally speaking, the filament experiences both radial and poloidal displacement, with the radial displacement on the outboard side of a toroidal device directed towards the wall, while the poloidal displacement can be directed "up" or "down" depending on the position of the mid-point of the filament and the magnitude of the shear.

As is well kown, when the filament is in contact with a conducting surface of the poloidal limiter, its motion is determined by a sheath resistance (e.g., [6.10]). We provide an averaging procedure that allows one to evaluate the contribution of this effect to the average motion of the filament.

For a long-enough filament (or cold-enough plasma), the resistive ballooning becomes possible and the filament, in the course of its motion, deviates from the field 
lines. We present a procedure that allows one to describe this motion in an arbitrary toroidally-symmetric field, for a filament of an arbitrary length.

\section{ACKNOWLEDGMENTS}

The author is grateful to R.H. Cohen, T.D. Rognlien, M.V. Umanski, A. Xiong, and $\mathrm{X} . \mathrm{Xu}$ for their interest and helpful comments. This work was performed under the auspices of the U.S. Department of Energy by University of California Lawrence Livermore National Laboratory under contract No. W-7405-Eng-48. 


\section{APPENDIX I The geometry of the flux tube}

The geodesic displacement in an arbitrary point is a linear combination of $\xi_{g 0}$ and $\xi_{n 0}$, the latter contribution being associated with the magnetic shear. We first evaluate the contribution of the geodesic displacement. It can be found from the observation that any field line on the given flux surface can be obtained from another field line on the same surface by toroidal rotation by some appropriate angle $\alpha$., Fig. 4. Simple geometrical considerations show that $\alpha R=\xi_{g}\left(B / B_{p} R\right)$. As $\alpha$ is constant along the given field line, we obtain:

$\xi_{g}=\xi_{g 0}\left(\frac{B_{p} R}{B}\right)\left(\frac{B_{0}}{B_{p 0} R_{0}}\right)$

To evaluate the contribution of the normal displacement, we note that the toroidal angle $\Delta \theta$ between the reference point and any other point at a given field line can be expressed as

$$
\Delta \theta=\int \frac{B_{T} d l}{R B_{P}}
$$

where the integration is taken along the flux surface from the reference point to a chosen point on the field line. For a given reference point, this is a function of the poloidal arc length and the magnetic surface (i.e., the poloidal magnetic flux $\psi$ ). We want to know, by how much the field line displaces in the poloidal direction, $\delta l \equiv \xi_{p}=\xi_{g} B / B_{T}$, in some cross-section characterized by the angle $\Delta \theta$ if we displace the foot point of the field line in the reference point by $\xi_{n 0}$ in the normal direction. For $\Delta \theta(l, \theta)=$ const, one has: $\delta l(\partial \Delta \theta / \partial l)+\delta \psi(\partial \Delta \theta / \partial \psi)=0, \quad \partial \Delta \theta / \partial l=B_{T} / R B_{P}, \quad \delta \psi=2 \pi R_{0} B_{P 0} \xi_{n 0}, \quad$ and $\xi_{g}=-\xi_{n 0} \frac{2 \pi R_{0} B_{p 0} R B_{P}}{B} \frac{\partial \Delta \theta}{\partial \psi}$ 
By summing up the contributions (A.1) and (A.3), we obtain:

$\xi_{g}=\left(\xi_{g 0}+Q \xi_{n 0}\right)\left(\frac{B_{p} R}{B}\right)\left(\frac{B_{0}}{B_{p 0} R_{0}}\right)$,

where the quantity

$Q=\left(\frac{2 \pi R_{0} B_{p 0}^{2}}{B_{0}}\right) \frac{\partial \Delta \theta}{\partial \psi}$

can be called "an average shear" 


\section{References}

[1] J.L. Terry, S.J. Zweben, K. Hallatschek, B. LaBombard, R.J. Maqueda, B. Bai, C.J.

Boswell, M. Greenwald, D. Kopon, W.M. Nevins, C.S. Pitcher, B.N. Rogers, D.P.

Stotler, X.Q. Xu. Phys. Plasmas, 10, 1739 (2003).

[2] G. Grulke, J.L. Terry, B. LaBombard, S. J. Zweben. Phys. Plasmas, 13, 012306 (2006).

[3] S.J. Zweben, R.J. Maqueda, J.L. Terry, T. Munsat, J.R. Myra, D. D’Ippolito, D.A.

Russell, J.A. Krommes, B. LeBlanc, T. Stolzfus-Dueck, D.P. Stotler, K.M. Wiliams, C.E. bush, R. Maingi, O. Grulke, S.A. Sabbagh, A.E. White. Phys. Plasmas, 13, 056114 (2006).

[4] S.I. Krasheninnikov. Phys. Lett. A 283, 368 (2001).

[5] S.C.Cowley, H. Wilson, O. Hurricane, B. Fong. Plasma Physics and Controlled Fusion, 45, A31, 2003; S.C. Cowley, H.R. Wilson, Physical Review Letters, 92, $1750061,2004$.

[6] S.I. Krasheninnikov, D.D. Ryutov, G. Yu. Journal of Plasma and Fusion Research, 6, 139 (2004).

[7] D.D. Ryutov, R.H. Cohen. Contributions to Plasma Physics, 44, 168 (2004).

[8] R.H. Cohen, D.D. Ryutov. Contrib. Plasm. Phys., 46, 678 (2006).

[9] D.A. D’Ippolito, J.R. Myra, S.I. Krasheninnikov. Phys. Plasmas, 9, 222 (2002).

[10] O.E. Garcia, N.H. Bian, W. Fundamentski. Phys. Plasmas, 13, 082309 (2006).

[11] S.I. Krasheninnikov, A.I. Smolyakov, G. Yu, T.K. Soboleva. Phys. Scr., T124, 13 (2006). 
[12] B.B. Kadomtsev. In: "Reviews of Plasma Physics," vol.2, M.A. Leontovich, Ed., (New York, Consultants Bureau), p. 153.

[13] G. Bateman. "MHD instabilities," Cambridge, MIT Press, 1978.

[14] W. Kunkel, J. Guillory. In: "Phenomena in Ionized Gases" (Proc. 7th Conf. Belgrade, 1965) Vol. 2, p. 702, Belgrade, 1966.

[15] P. Stangeby. The plasma boundary of magnetic fusion devices. (IoP Publishing, Bristol, 2000), p. 81.

[16] H.Berk, D.D.Ryutov, Yu. A.Tsidulko. "Temperature-gradient instability caused in plasma by conducting end-surfaces". JETP Lett., v.52, p.23 (1990). 


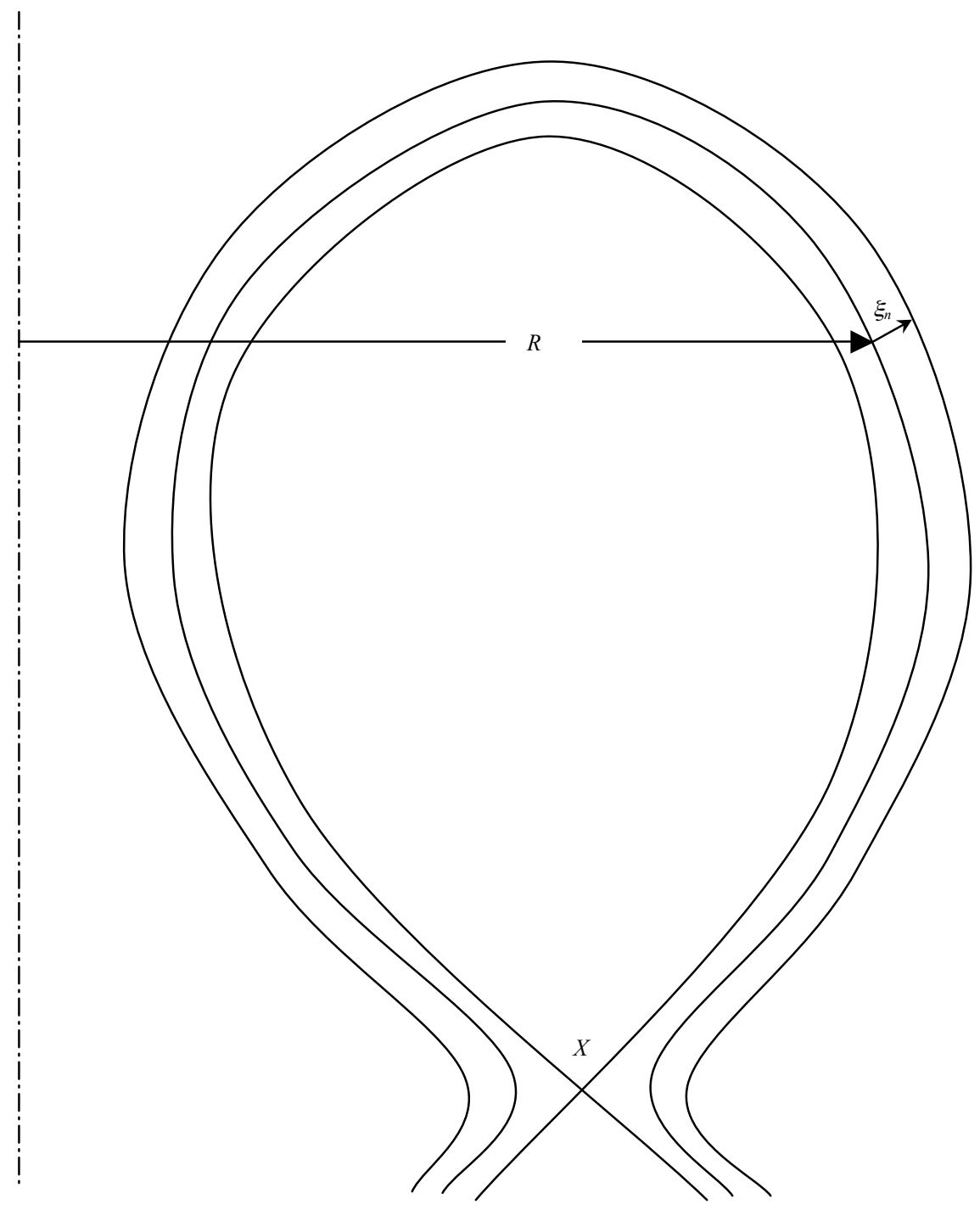

Fig. 1 Poloidal cross-section of a tokamak. Dash-doted line at the left is a major axis, $\mathrm{R}$ is a major radius for a given point on the flield line, $\xi_{\mathrm{n}}$ is a normal displacement 

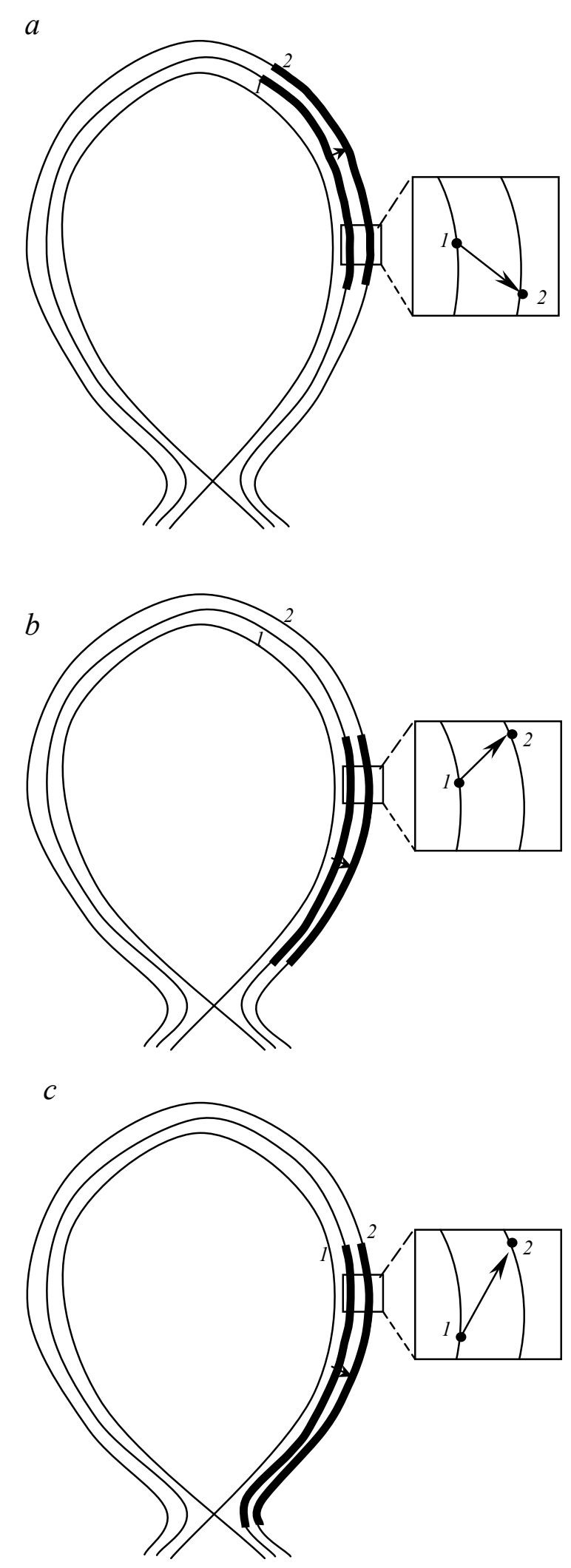

Fig. 2. Various geometries of filaments 


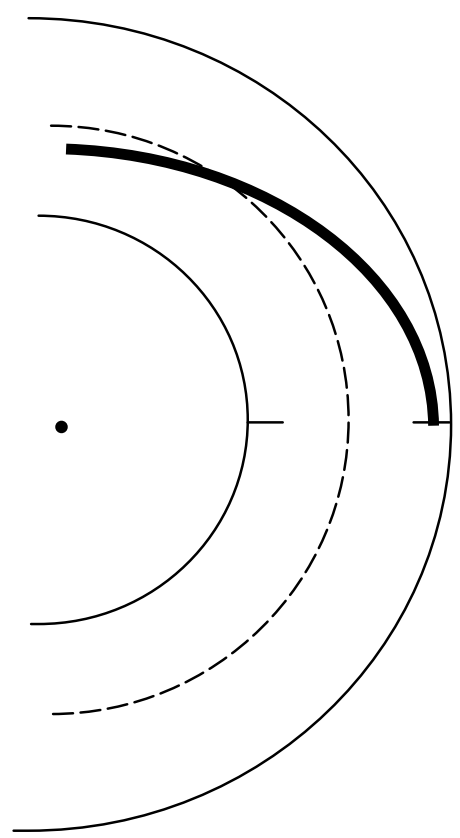

Fig. 3 projection of the filament (fat line) on the equatorial plane. The limiter is illustrated by two short lines. The magnetic axis is a dashed line. 


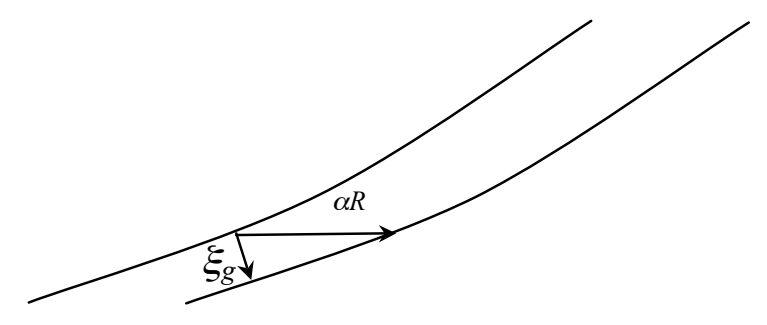

Fig. 4. Relating geodesic and toroidal displacement of the field line on the same flux surface. 